\title{
Buffer Rod Design for Measurement of Specific Gravity in the Processing of Industrial Food Batters
}

\author{
Fox, Paul D.; Smith, Penny Probert
}

Published in:

Proceedings IEEE Ultrason. Symp.

Publication date:

2002

Document Version

Publisher's PDF, also known as Version of record

Link back to DTU Orbit

Citation $(A P A)$ :

Fox, P. D., \& Smith, P. P. (2002). Buffer Rod Design for Measurement of Specific Gravity in the Processing of Industrial Food Batters. In Proceedings IEEE Ultrason. Symp. (pp. 660-663). IEEE.

\section{General rights}

Copyright and moral rights for the publications made accessible in the public portal are retained by the authors and/or other copyright owners and it is a condition of accessing publications that users recognise and abide by the legal requirements associated with these rights.

- Users may download and print one copy of any publication from the public portal for the purpose of private study or research.

- You may not further distribute the material or use it for any profit-making activity or commercial gain

- You may freely distribute the URL identifying the publication in the public portal

If you believe that this document breaches copyright please contact us providing details, and we will remove access to the work immediately and investigate your claim. 


\title{
Buffer Rod Design for Measurement of Specific Gravity in the Processing of Industrial Food Batters
}

\author{
Paul D Fox* $\quad$ Penny Probert Smith ${ }^{\dagger} \quad$ Sarabjit S Sahi ${ }^{\ddagger}$
}

\begin{abstract}
A low cost perspex buffer rod design for the measurement of specific gravity during the processing of industrial food batters is reported. Operation was conducted in pulsed mode using a $2.25 \mathrm{MHz}, 15 \mathrm{~mm}$ diameter transducer and the intensity and an analytic calibration curve relating buffer rod output to specific gravity is obtained. The probe design may have application to other similar mixtures or industrial sludges in which similar material properties are observed.
\end{abstract}

\section{INTRODUCTION}

In food batters, the incorporation of air in a finely dispersed form has an important influence on its properties, including appearance, texture, consistency and size per unit weight. In fact the presence of a well-defined volume of gas cells can be essential for the characteristic properties of that particular food [1]. In this study we concentrate on high ratio industrial cake batters consisting of cake flour, sugar, fat, milk powder, salt, egg, glycerine and water. The amount of air beaten into in these batters during the mixing process is reflected in a change of specific gravity of the batter and determines to a large extent the overall quality of the cake [2], as well as affecting cake volume [3] and rheological flow properties [4]. The specific gravity of the batter varies as a function of mixing time, and here we focus on the initial drop in specific gravity since this is the phase during which mixing is terminated in the industrial mixers. The traditional method for establishing the stopping time is

${ }^{*}$ Center for Fast Ultrasound Imaging, Ørsted-DTU, Building 348, Technical University of Denmark, DK-2800 Kgs. Lyngby, Denmark. (pdf@oersted.dtu.dk)

${ }^{\dagger}$ Robotics Research Group, Department of Engineering Science, University of Oxford, Parks Road, Oxford OX1 3PJ, UK. (pjp@ robots.ox.ac.uk)

${ }^{\ddagger}$ Campden \& Chorleywood Food Research Association, Chipping Campden, GL55 6LD, UK. (s.sahi@ campden.co.uk)
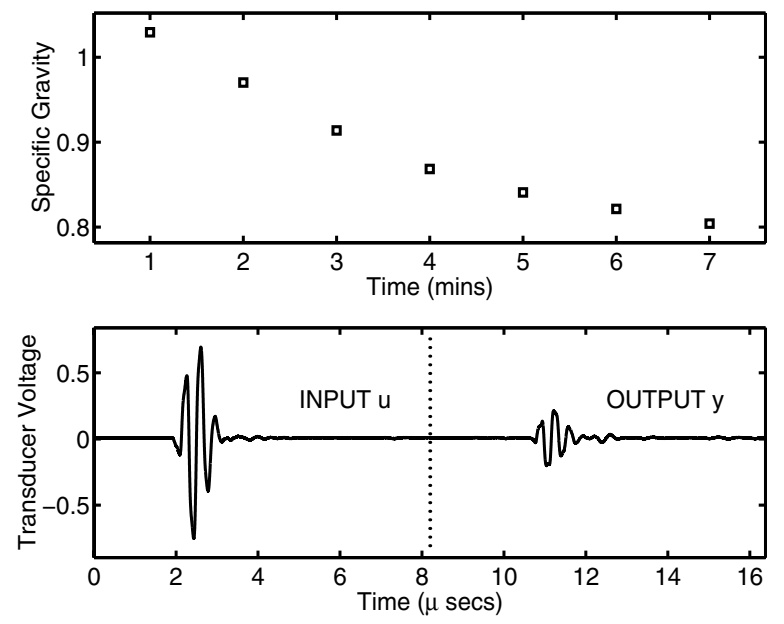

Figure 1: Upper plot : Manually measured specific gravity versus time for Mix 1. Lower plot: Typical input-output transducer voltage time series at a given measuring instant. Left time window is considered as input signal and right window as output signal.

to halt the process periodically, remove and manually weigh a batter sample of fixed volume in order to determine its specific gravity $\left(S_{b}\right)$. This is a time consuming and labor intensive process, which ideally could be replaced by an automated ultrasonic device online without human intervention. In this article we report a low cost perspex buffer rod design for this purpose.

Prior to mixing, all ingredients are originally equilibrated to $21^{\circ} \mathrm{C}$ prior to mixing and slowly blended together at a low mixing speed for an initial period of 90 seconds in order to achieve batter homogeneity. The measured mixing then takes place in blocks of $60 \mathrm{sec}-$ ond periods, and the batter density is measured by determining the weight of batter within a container of known volume (Figure 1, upper). At each point in time, the probe is also inserted into the batter, with the probe out- 
put voltage being a time series of the form of Figure 1 (lower). The objective is then to deduce an ultrasonic measure of the specific gravity which can provide a calibration for future use of the probe without the need to measure specific gravity manually.

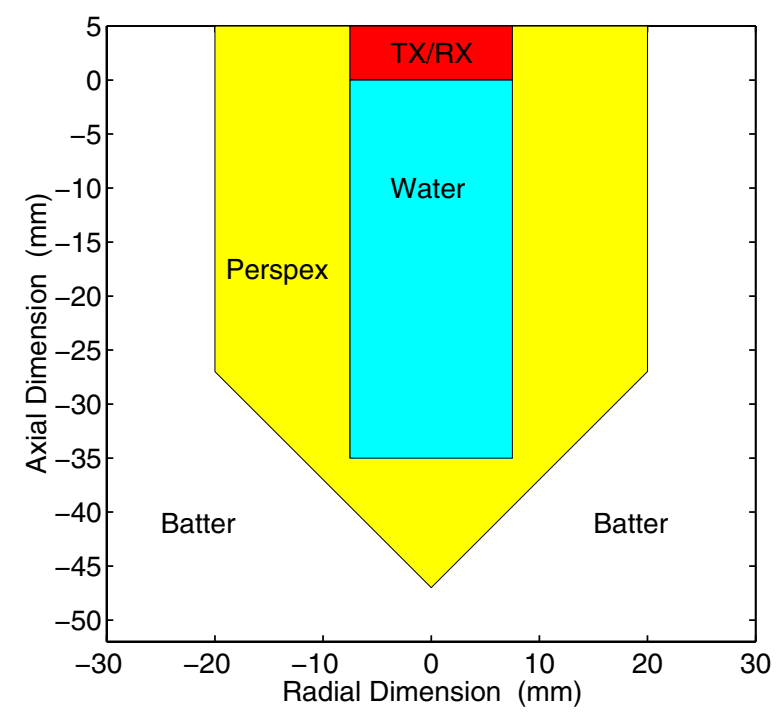

Figure 2: Schematic cut through probe centerline.

\section{PROBE ANALYSIS}

Construction of the probe was as per Figure 2. This simple low-cost design consists of a perspex outer chamber housing an internal $2.25 \mathrm{MHz} 15 \mathrm{~mm}$ diameter transducer (TX/RX) and water chamber, which are separated from the batter by means of the perspex housing. This housing is terminated in the form of a $45^{\circ}$ conical tip such that it enters the batter cleanly without trapping any external air bubbles on the outer surface as it enters into the batter sample. To analyse the probe behaviour, we use the standard model for a plane wave passing from the first medium (' $f$ ') to the second medium (' $s$ ') with the following reflection and transmission coefficients $R_{f s}$ and $T_{f s}$ :

$$
\begin{aligned}
R_{f s} & =\frac{\rho_{s} c_{s} \cos \theta_{f s}-\rho_{f} c_{f} \cos \phi_{f s}}{\rho_{s} c_{s} \cos \theta_{f s}+\rho_{f} c_{f} \cos \phi_{f s}} \\
T_{f s} & =\frac{2 \rho_{s} c_{s} \cos \theta_{f s}}{\rho_{s} c_{s} \cos \theta_{f s}+\rho_{f} c_{f} \cos \phi_{f s}}
\end{aligned}
$$

Here $\rho_{f}, \rho_{s}$ and $c_{f}, c_{s}$ are the densities and velocities for media $f$ and $s$ respectively. The angles $\theta_{f s}$ are the incident impact/reflection angle in medium $f$ and $\phi_{f s}$ is the propagation angle into medium $s$. Snell's law also relates $\phi_{f s}$ to $\theta_{f s}$ via the velocities $c_{f}, c_{s}$ as $\sin \phi_{f s}=$ $\left(c_{s} \sin \theta_{f s}\right) / c_{f}$. Then, neglecting any losses in the water and perspex, the following reflection-transmission sequence (summarised in Table 1) is assumed. Firstly a

Table 1: Summary of Reflection and Transmission Sequences

\begin{tabular}{c|c|c}
$\begin{array}{c}\text { Interface } \\
\text { Sequence }\end{array}$ & $\begin{array}{c}\text { Reflected } \\
\text { Pressure }\end{array}$ & $\begin{array}{c}\text { Transmitted } \\
\text { Pressure }\end{array}$ \\
\hline 1. Water-Perspex & $R_{w p} P$ & $T_{w p} P$ \\
2. Perspex-Batter & $R_{p b} T_{w p} P$ & $T_{p b} T_{w p} P$ \\
3. Perspex-Batter & $R_{p b}^{2} T_{w p} P$ & $T_{p b} R_{p b} T_{w p} P$ \\
4. Perspex-Water & $R_{p w} R_{p b}^{2} T_{w p} P$ & $T_{p w} R_{p b}^{2} T_{w p} P$
\end{tabular}

pulsed wave of instantaneous amplitude $P$ travels down the water channel and impacts upon the water-perspex interface. An amount $R_{w p} P$ is then reflected back to the transducer giving rise to a measured ('input') voltage $u=H R_{w p} P$ where $H$ is the transducer transfer function at the frequency of interest. A pressure $T_{w p} P$ is transmitted through the interface into the perspex and then impacts against the $45^{\circ}$ perspex-batter interface. A pressure $R_{p b} T_{w p} P$ is then reflected back into the perspex whilst a pressure $T_{p b} T_{w p} P$ transmitted into the batter at an unknown transmission angle $\phi_{p b}$. The reflected pressure $R_{p b} T_{w p} P$ then travels through the perspex across to the other tip face, where the same perspexbatter interaction takes place once again. This gives a reflected pressure $R_{p b}^{2} T_{w p} P$ into the perspex and transmitted pressure $T_{p b} R_{p b} T_{w p} P$ into the batter. Finally the wave of pressure $R_{p b}^{2} T_{w p} P$ impacts the perspexwater interface, with pressure $R_{p w} R_{p b}^{2} T_{w p} P$ being reflected back into the perspex and pressure $T_{p w} R_{p b}^{2} T_{w p} P$ being transmitted through into the water channel.The transmitted wave travels to the transducer surface and gives rise to a measured ('output') voltage of $y=$ $H T_{p w} R_{p b}^{2} T_{w p} P$. The probe gain $G$ at a given frequency is then defined as the ratio of output $y$ to input $u$

$$
G=\frac{T_{p w} R_{p b}^{2} T_{w p}}{R_{w p}}
$$

for which the component terms are listed in Table 2. 
Table 2: Known and unknown process parameters

\begin{tabular}{c|c|c}
\hline \hline Medium & Density $\left(\frac{k g}{m^{3}}\right)$ & Velocity $\left(\frac{m}{s}\right)$ \\
\hline Water & $\rho_{w}=1000$ & $c_{w}=1480$ \\
Perspex & $\rho_{p}=1176$ & $c_{p}=2730$ \\
Batter & $\rho_{b}=?$ & $c_{b}=?$ \\
& & \\
\hline \hline Interface & Ancidence/Reflection & Transmission \\
Water-Perspex & $\theta_{w p}=0$ & $\phi_{w p}=0$ \\
Perspex-Water & $\theta_{p w}=0$ & $\phi_{p w}=0$ \\
Perspex-Batter & $\theta_{p b}=45$ & $\phi_{p b}=?$ \\
\hline \hline Interface & $\gamma\left(\times 10^{-7} \frac{m^{2} s}{k g}\right)$ & $\delta\left(\times 10^{-4} \frac{s}{m}\right)$ \\
\hline Water-Perspex & $\gamma_{w p}=6.7568$ & $\delta_{w p}=3.6630$ \\
Perspex-Water & $\gamma_{p w}=3.1148$ & $\delta_{p w}=6.7568$ \\
Perspex-Batter & $\gamma_{p b}=2.2025$ & $\delta_{p b}=?$ \\
& & \\
\hline \hline & Reflection & Transmission \\
Interface & Coefficient $R$ & Coefficient $T$ \\
\hline Water-Perspex & $R_{w p}=0.3689$ & $T_{w p}=1.3689$ \\
Perspex-Water & $R_{p w}=-0.3689$ & $T_{p w}=0.6311$ \\
Perspex-Batter & $R_{p b}=?$ & $T_{p b}=?$ \\
& &
\end{tabular}

Notice that the reflection coefficient $R_{p b}$ for the interface between perspex and batter is unknown. Notice also that the gain $G$ in equation (3) general varies as a function of both pulse component frequency and mixing time. Figure 3 (upper) shows this behaviour, in which the lower line represents the gain at different frequencies after one minute of mixing (time $t_{1}$ ) and the upper line represents the gain after 7 minutes of mixing (time $t_{7}$ ). For the remaining analysis we then choose to operate at a given frequency, in which a component just over $2 \mathrm{MHz}$ was found to give the most reliable results in practice. Figure 3 (lower) shows gain at this frequency against mixing time, with the experimentally measured relationship between gain and specific being given in Figure 4 (upper). Substituting for $R_{p b}$ from (1) by replacing subscripts ' $f$ 'and ' $s$ 'with ' $p$ ' and ' $b$ ' for perspex and batter respectively to the batter specific gravity $S_{b}=\rho_{b} / \rho_{w}$ as
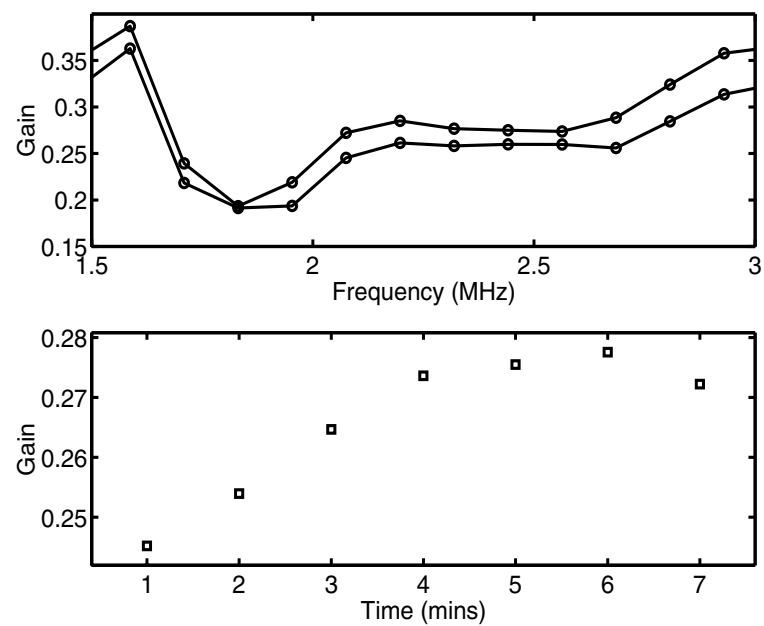

Figure 3: Upper plot: Gain magnitudes vs frequency for Mix 1 at times $t_{1}$ (lower line) and $t_{7}$ (upper line). Lower plot: Transducer gain $G$ at $2.0752 \mathrm{MHz}$ versus time for Mix 1.

$$
S_{b}=\delta_{p b} \times \frac{1+K \sqrt{G}}{\gamma_{p b} \rho_{w}(1-K \sqrt{G})} \quad: \quad \delta_{p b}=\frac{\cos \phi_{p b}}{c_{b}}
$$

where $\gamma_{p b}=\left(\cos \theta_{p b}\right) / \rho_{p} c_{p}$ and $K=\sqrt{R_{w p} / T_{w p} T_{p w}}$ and $\rho_{w}$ are known but $\delta_{p b}$ is unknown since both $c_{b}$ and $\phi_{p b}$ are unknown. Hence $S_{b}$ cannot be obtained in practice from equation (4) until a reasonable estimate of $\delta_{p b}$ is obtained, achievable by rearranging (6) to give

$$
\delta_{p b}=S_{b} \times \frac{\gamma_{p b} \rho_{w}(1-K \sqrt{G})}{1+K \sqrt{G}}
$$

Note then that the right hand side of this equation may then be plotted from the measured values of gain $G$ and specific gravity $S_{b}$ for a given 'calibration' data set (Lower plot, Figure 4). This function may be well approximated in by a straight line of the form $\delta_{p b} \approx \alpha+$ $\beta S_{b}$ in the region of interest (where $\alpha=7.5597 \times 10^{-5}$ and $\beta=3.5898 \times 10^{-4}$ for a least squares fit). Substituting for $\delta_{p b}$ into equation (5) and rearranging then gives

$$
\alpha+\beta S_{b} \approx S_{b} \times \frac{\gamma_{p b} \rho_{w}(1-K \sqrt{G})}{1+K \sqrt{G}}
$$

from which we obtain a final calibrated estimate of the specific gravity for subsequent batter samples as 

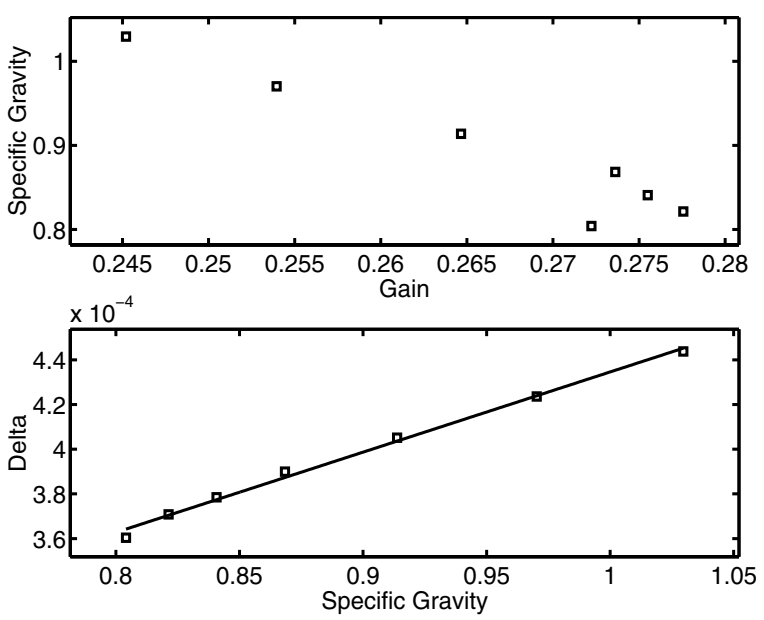

Figure 4: Upper plot: Specific gravity versus gain for Mix 1. Lower plot: Delta versus specific gravity for Mix 1.

$$
\hat{S}_{b}=\frac{\alpha}{\gamma_{p b} \rho_{w}\left(\frac{1-K \sqrt{G}}{1+K \sqrt{G}}\right)-\beta}
$$

where $\hat{S}_{b}$ represents the estimate of $S_{b}$ obtained by removing the approximation sign from (6). Figure 5 (upper) shows the resulting $\hat{S}_{b}$ calibration curve obtained from Mix 1, and Figure 5 (lower) then shows estimates $\hat{S}_{b}$ for a second mix, in which the $\alpha$ and $\beta$ parameters from Mix 1 have been used to generate the estimates for the second Mix from equation (7). In both cases the estimated specific gravities fit the measured data well.

\section{CONCLUSIONS}

The buffer rod proved successful as a technique to measure properties at the interface to a high viscosity, air filled mixture which did not support significant transmission. The general design may also have application to measurement of specific gravity in a wider array of industrial substances.

\section{ACKNOWLEDGMENTS}

This work was sponsored principally by DEFRA Bridge Link Grant FQS-21 in the United Kingdom. The authors are grateful to Dave Cartwright from Cygnus In-
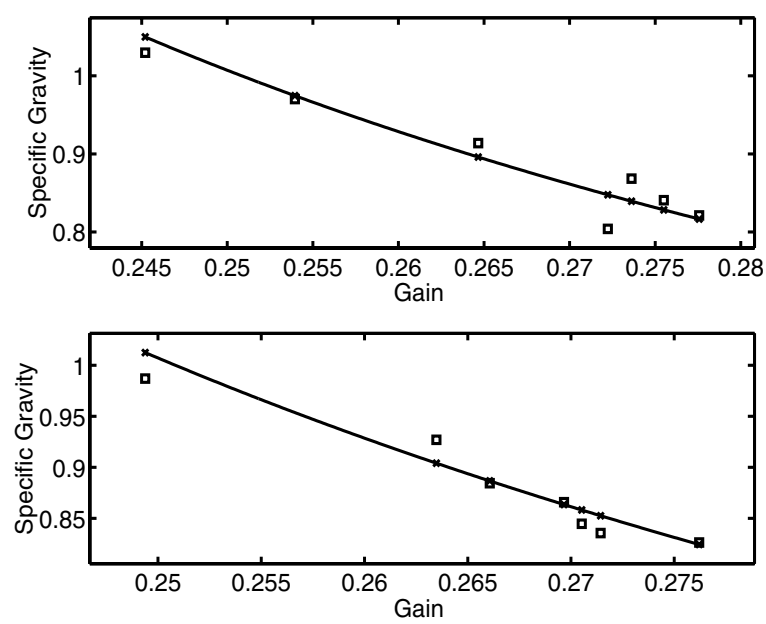

Figure 5: Upper plot: Measured and calibrated specific gravities versus gain for Mix 1. Lower plot: Estimated and measured specific gravity for Mix 2.

struments Ltd, Dorchester, UK for his initial suggestion to try a buffer rod approach and for the loan of the probe transducer. Also to Maurice Keeble-Smith, Ken Howson and John Hastings at Oxford University for assistance with construction of the probe, and Paul Catterall and Dr Terry Sharp at Campden \& Chorleywood Food Research Association for their assistance with experimental procedures. Final data analysis was completed under grant 26-01-0178 from the Danish Science Foundation, Denmark

\section{$\mathrm{V}$ REFERENCES}

[1] J. W. Mansvelt. The use of foams in foods and food production. Foams, Proceedings of the Colloid and Surface Chemistry Group of the Society of Chemical Industry, Brunel University, pages 261-274, 1975.

[2] G. T. Carlin. A microscopic study of the behaviour of fats in cake batters. Cereal Chemistry, 21:189-199, 1944.

[3] D. G. Hodge, R. J. Woodward, and P. Wade. Control of batter specific gravity. Baking Industry Journal, 3:2638, 1972.

[4] S. S. Sahi. Influence of aeration and emulsifiers on cake batter rheology and textural properties of cakes, pages 263-271. Bubbles in Foods, American Association of Cereal Chemists. St Paul, Minnesota, USA., 1999. Eds. G. M. Campbell and C. Webb and S. S. Panediella and K. Niranjan. 